\title{
MULTI-SCALE AND SCALE DIMENSION PROPERTIES IN SPATIAL RASTER MODELLING - CONCEPT AND CURRENT IMPLEMENTATION
}

\author{
Hairi Karim ${ }^{1,2}$, Alias Abdul Rahman ${ }^{1}$, Mohd Radhie bin Mohd Salleh ${ }^{2}$. \\ ${ }^{1} 3 \mathrm{D}$ GIS Research Lab, \\ Faculty of Build Environment and Survey, \\ Universiti Teknologi Malaysia, \\ 81310, Johor Bahru, Johor, Malaysia. \\ wmhairigis@gmail.com and alias@utm.my \\ ${ }^{2}$ I Net Spatial Sdn Bhd, \\ Taman Pulai Utama, 81300 Skudai, \\ Johor, Malaysia. \\ wmhairi@inetspatial.com and mradhie@inetspatial.com
}

KEY WORDS: Multi-scale, raster scale dimension, raster pixel resolution, raster analysis, resampling raster resolution.

\begin{abstract}
:
Various users and applications required different abstraction details of spatial model either in vector or/and raster data types/models. Generating different model abstraction details (e.g. Level of Detail/LOD) produces various drawbacks especially for data model sharing among stakeholders or publics. Different abstraction detail or LOD means different details in geometry, semantic information, attributes as well as different accuracy provided within the vector model (e.g. a certain LOD). On the other hand, raster dataset with different resolutions on certain information or layer (e.g. elevation, land cover, spatial imagery, soil type, thematic raster map and others) could also be considered as multi-scale raster modelling which produces similar drawbacks with additional storage redundancy/consumption and updating works. There are some solutions for vector scale modelling such as CityGML (3D) and multiscale or vario-scale (2D) modelling induce good solutions for vector; however, there are no solution for raster data type (or model) yet. Thus, a concept description in categorizing and defining multi-scale for multi-resolution raster dataset should be introduced. This paper basically highlights the similarity of spatial 2D vector and raster type GIS dataset, some introduction and properties of raster dataset which able to be defined it as the same level of vector LoD in scale modelling. This paper basically tries to kick off a new multi-scale domain in supporting spatial raster dataset (new idea), which will be then be extend/expand by related researchers near the future. Discussion on successful implementation of vector multi-scale model will be in the paper as well as existing multiscale approach in storing raster dataset as the main content of the paper. Some potential analysis on related multi-scale raster and validation are also discussed to give brief idea on what is spatial raster capable of; especially to those who are new/not yet engage with this multi-scale spatial raster dataset.
\end{abstract}

\section{INTRODUCTION}

Different applications or users need different abstractions and information details of the real-world phenomenon (Karim and et al., 2016); in other word, different models of a particular 2D/3D object. Each application requires its own set level of details (LoDs) to be embedded into the model abstraction. Most of the models focus on the geometry accuracy, attribute and some semantic information (visualization and measurement purposes). However, less emphasis was given on raster multiscale dimension modelling.

The paper discusses brief explanation of vector and raster GIS data type/model for the integration with scale dimension. Section 2 explains some concepts and implementation literatures on scale dimension for both vector and raster GIS dataset. Section 3 discusses on conceptual scale dimension for raster and current implementation as in term of storage aspect. Multi-scale raster analysis and validation are described in section 4 while conclusion of the paper in the last section.

\subsection{Spatial Data Type}

As GIS spatial dataset is concerned, there are two main categories (types) of GIS spatial data; vector and raster as in Figure 1 and Figure 2 below.

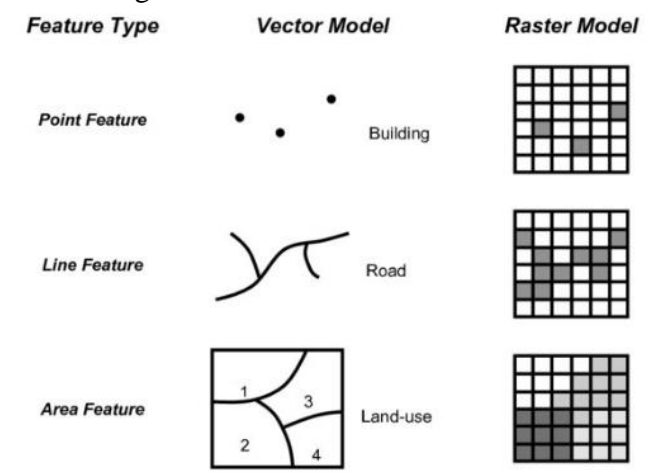

Figure 1. Illustration of vector and raster spatial data type (Zhu, 2014) 


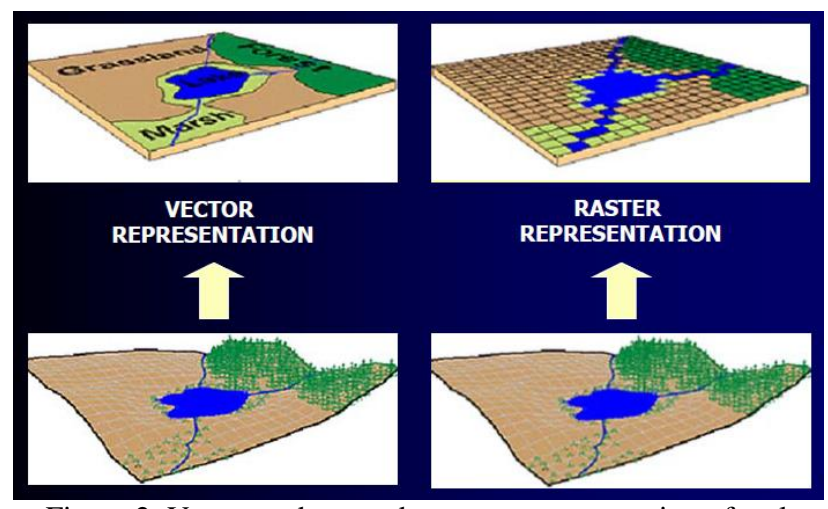

Figure 2. Vector and raster data type as presentation of real world features / map. (Pramoda Raj, 2017)

In general, spatial raster data type consumes more storage space as compared to vector data type of the same extend (sample area - except vector data carries plenty of attributes information or large scale vector map). However, raster data type have it own advantage especially with faster computational time as compared to vector data type (Pathan, 2012) using same hardware specifications.

\subsubsection{Vectors}

Vector (also known as object-oriented feature) is a coordinatebased data model which made up to store geometry primitives (such as point, line and polygon) and the object attributes.

Point vector is simply XY coordinate (latitude-longitude or north-easting based spatial referenced system). Lines usually represent features that are linear in nature; stored as a sequence of XY points. For example, maps show rivers, roads and pipelines as vector lines. While, a polygon is a set of connected vertices (XY points) in a particular order and the coordinate of starting point is equal to the end point (closed barrier).

\subsubsection{Raster}

The term raster implies for regular spaced grid - consists of rows and column of pixels. Raster uses a single value to represent a multiplicity of parameters e.g. elevation and others. The second types of raster category is digital images which support combination of three or more values such as $\mathrm{RGB} /$ multiple bands (refers to Table 1).

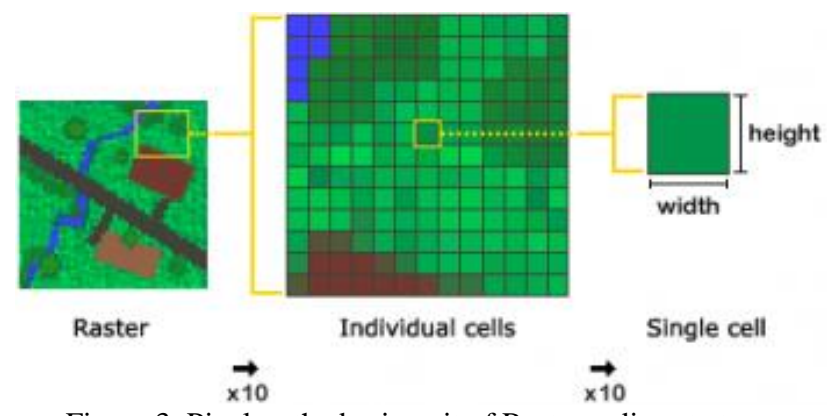

Figure 3. Pixel as the basic unit of Raster - discrete type (GICHD, 2016)

Raster can be categorised into two main groups; discrete and continue. An example of discrete is a grid represents of land cover class/soil type (unconnected/non-relational neighbouring objects) while Digital Elevation/Terrain Model (DEM/DTM) is an example of continues raster (seamless, Figure 4).
Later, raster could be sub-divided into several groups include but not limited to; DEM, satellite imageries, orthophotos, binary scanned files, graphics files, chloropleth scanned maps, attribute based raster (e.g. land use, land covers) non-spatial dimension parameters (e.g. temperature, time stamp/video recording etc) and others.

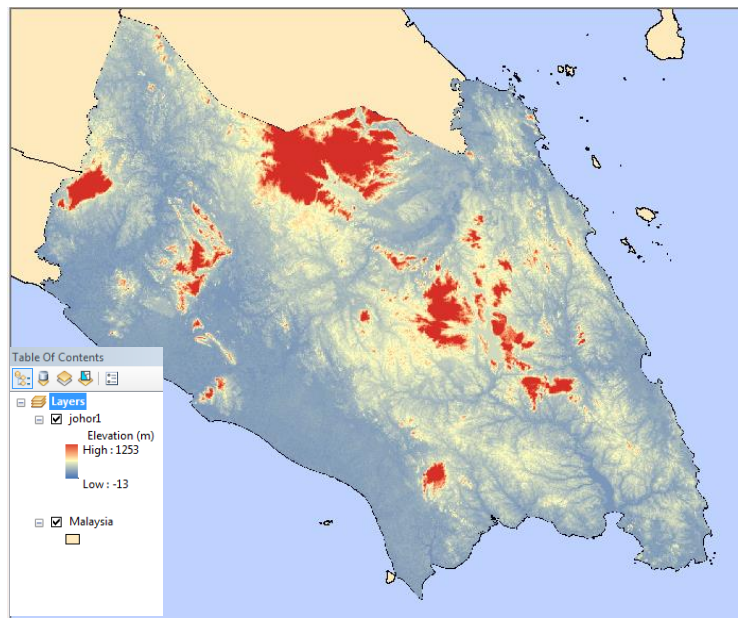

Figure 4. Continues type of raster (e.g. DEM)

\section{THE LITERATURE}

This section is divided into three sub-sections; multi-scale modelling which will later expanded into vector and raster scale dimension.

\subsection{Multi-scale Modelling (General)}

Traditionally, 2D multi-scale objects are stored and structured separately in different databases and viewers. This implementation approach resulting some drawbacks in finding/searching information from other detailed objects lack of relationships, limited queries, redundant in storage, updating works and others. Thus, a limited extracted information from the analysis or developed applications.

Different applications or/and users need different abstractions of the real-world phenomenon (Karim and et al., 2016); each application requires its own set level of details (LoD) or resolution to be embedded into their viewer/result (model/dataset). Most of the research and implementation focuses on multi scale of vector models; less emphasis was given for raster datasets.

\subsection{Vector Scale Dimension}

Current implementation frameworks in scale integration of 2D spatial data are either using the generalization techniques or storing the individual level of detail data into separate databases (multi-scale, Figure 5) or vario-scale approaches (Figure 6 and Figure 7). 


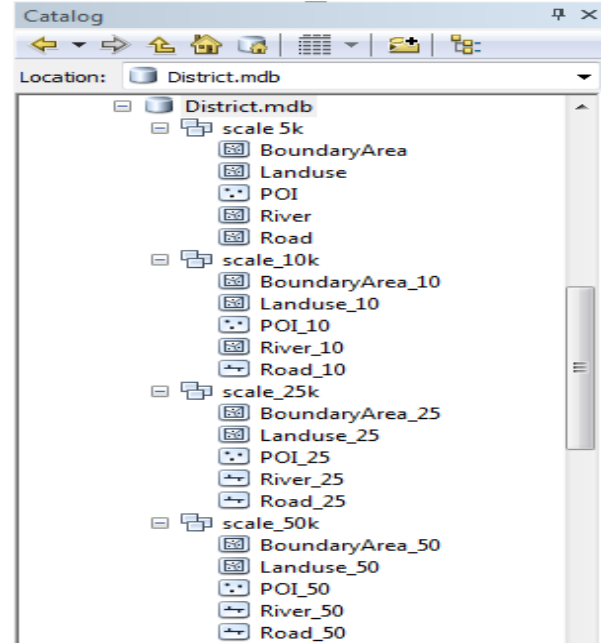

Figure 5. Multi-scale in separated database, with no connected topology/link between LoD and redundancy of attributes/geometry.

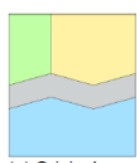

(a) Original map

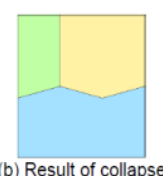

(b) Result of collapse

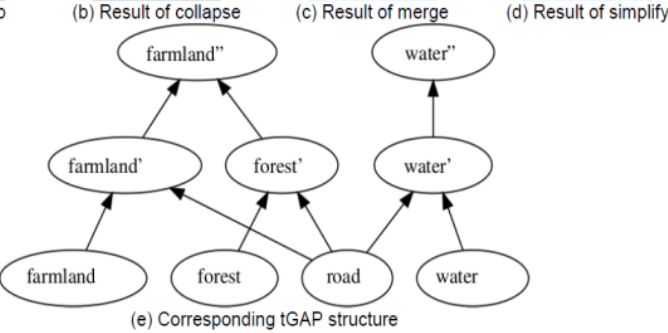

Figure 6. Vario-scale approach, topology connects hierarchical between LoD and good in storing attribute (Meijers, 2011).
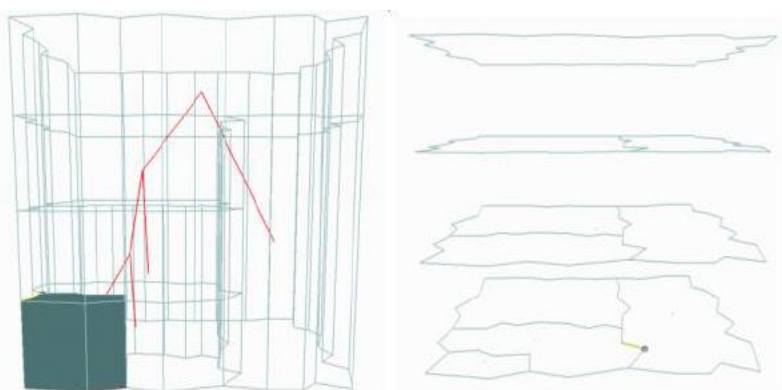

Figure 7. Accesssing n-LoD with dual graph (Karim and et al., 2016)

\subsection{Raster Scale Dimension}

Less research, reference and implementation which suggest raster of different resolution (pixel) considered raster as a scale dimension. However, the concept, output and storage implementation of raster dataset (same parameter such as elevation) are closely similar to multi-scale of vector dataset. This paper basically tries to convince the reader that raster either by different resolution and attribute is also could be defined and treated as within scale dimension (different detail levels -resolution and attribute as in Table 1).

\subsubsection{Raster Attribute Dimension}

Although in general and basic unit of raster only carry a single value (except it metric coordinate/reference), repetition of this raster layer by time (sequence of the same resolution and area with different time series) or attribute changes by geometry/pixel value is also consider as non-spatial dimension.

Table 1. Multi-scale dimension for raster

\begin{tabular}{|c|c|c|c|c|c|c|}
\hline \multirow{2}{*}{$\begin{array}{l}\text { Multi-Scale } \\
\text { (Resolution ) }\end{array}$} & \multicolumn{6}{|c|}{ Dimension / Attribute } \\
\hline & Elevation & Land Use & Land Cover & Temperature & Spectral & Others \\
\hline $2 \mathrm{~m} \times 2 \mathrm{~m}$ & \multirow{6}{*}{$\begin{array}{l}\text {-Time (e.g. years) } \\
\text {-Changes over } \\
\text { development/ } \\
\text { land use } \\
\text {-Sources of data }\end{array}$} & \multirow{6}{*}{$\begin{array}{l}\text {-Time (e.g. years) } \\
\text {-Changes over } \\
\text { development }\end{array}$} & \multirow{6}{*}{$\begin{array}{c}\text {-Time (e.g. years) } \\
\text {-Changes over } \\
\text { development }\end{array}$} & \multirow{6}{*}{$\begin{array}{l}\text {-Time } \\
\text { (e.g. years) } \\
\text {-Changes over } \\
\text { heat/time }\end{array}$} & \multirow{6}{*}{\begin{tabular}{|l|}
-Years \\
-Satellite/Drone \\
-Orthophoto \\
-Sources of data
\end{tabular}} & Soil type \\
\hline $4 \mathrm{~m} \times 4 \mathrm{~m}$ & & & & & & Scanned Map \\
\hline $12 \mathrm{~m} \times 12 \mathrm{~m}$ & & & & & & Pattern \\
\hline $24 \mathrm{~m} \times 24 \mathrm{~m}$ & & & & & & Distribution \\
\hline $30 \mathrm{~m} \times 30 \mathrm{~m}$ & & & & & & $\begin{array}{l}\text { Hotspot } \\
\text { crimes/diseases }\end{array}$ \\
\hline $90 \mathrm{~m} \times 90 \mathrm{~m}$ & & & & & & Others \\
\hline
\end{tabular}

\subsubsection{Raster Resolution}

At a given area, several different raster pixel resolution layers (clearer and blurred) are also considered as scale dimension parameter (different LoD in modelling the same spatial object/parameter). For example, 1 metre resolution of LiDAR dataset (Light Detection and Ranging), 5 metre resolution of IFSAR dataset (Interferometric Synthetic-Aperture Radar) and a 30 metre of SRTM dataset (Shuttle Radar Topography Mission) at the same area and year of data acquisition are an example of LoD in raster multi-scale (dimension) 


\section{MULTI-SCALE RASTER CONCEPT AND CURRENT IMPLEMENTATION}

This section discusses on how we actually may describe the conceptual and include raster with different resolutions as series of datasets in scale dimension. Current implementation of multiscale (multi-resolutions) spatial raster dataset for the same area is also discussed. Multi-resolution raster normally stored in file/folder based directory or in spatial database such as ArcGIS and PostgreSQL. There are two type of raster (Figure 8); discrete and continues as mentioned previously in section 1.1.2.

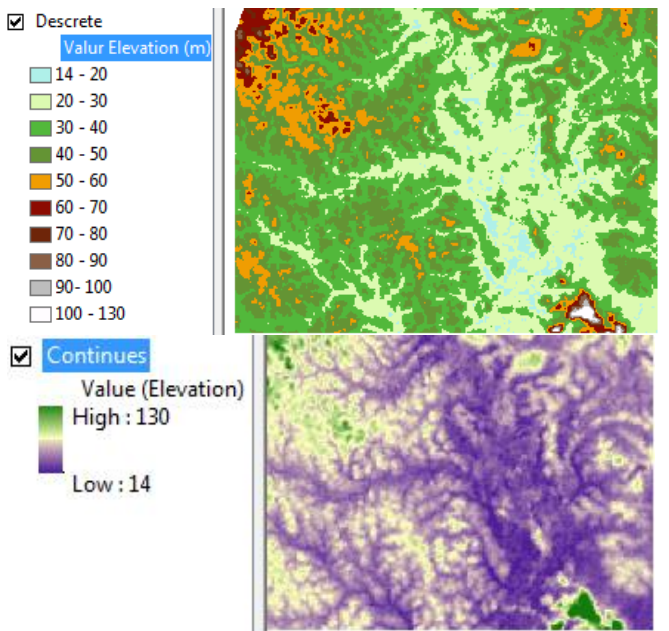

Figure 8. Type of spatial raster (a-descrete and b-continues)

\subsection{Multi-scale Raster Concept}

\subsubsection{Imagery / scanned documents (Discrete Type)}

Discrete raster is a type of raster which have a unit value independantly and did not have specific relationship/rule with neigbouring pixels (or a group of pixels represent a certain object such as tree, buildings, road and other). The sources of discrete raster normally from imagery, scanned maps, converted vector layer, true/false $(1,0)$ raster analysis result and others. It also sometime refers to a continues parameter (layer) which being classified in a few different classes/categories to diffenciate or represent information into several groups

For this example, a downloaded (screen captured) of Google Earth Pro images on a particular date (e.g. 19 May 2019) with different resolutions; Min 1366 x 672, HD 1920 x 1080 and Max 4800 x 2361 as shown in Figure 9a - 9c respectively. These are in standard image format (JPG) without spatial references (not yet considered as spatial image/raster GIS dataset). The storage cost for respective image resolutions are shown in Figure 10.

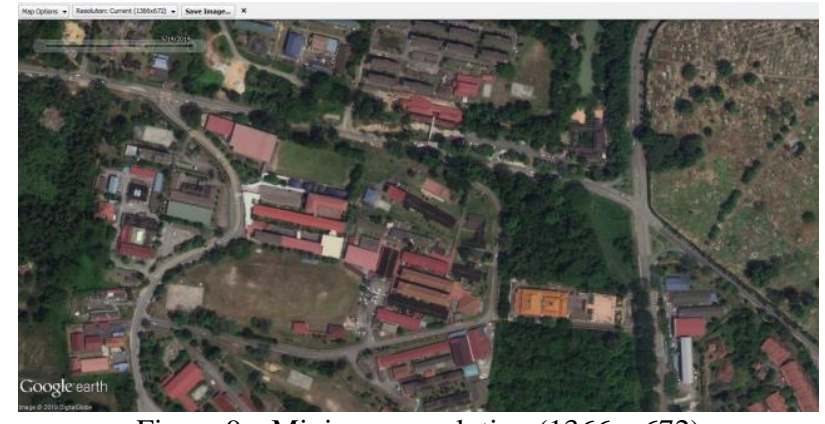

Figure 9a. Minimum resolution (1366 x 672)

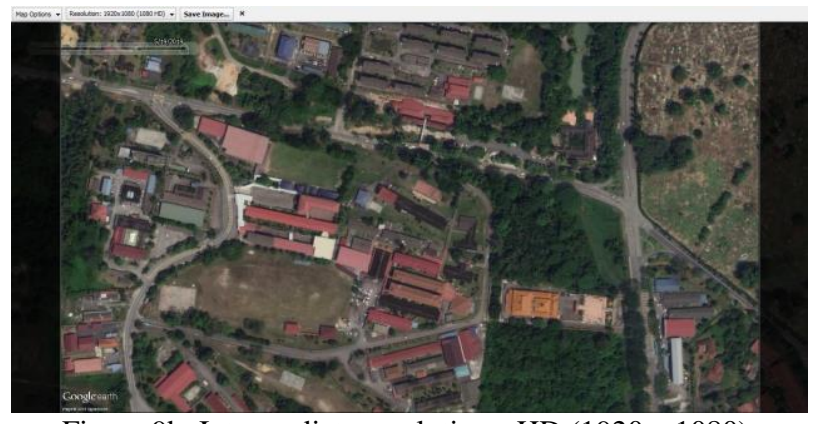

Figure 9b. Intermediate resolution - HD (1920 x 1080)

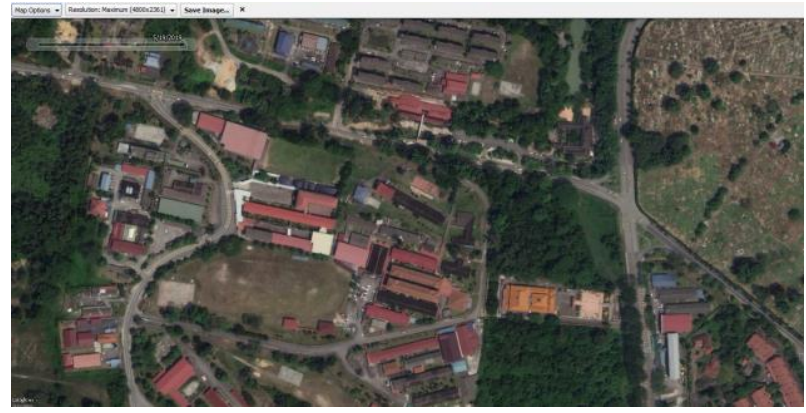

Figure 9c. Maximum resolution (4800 x 2361)

\begin{tabular}{lllr}
\hline 国 GE_1366x672.jpg & $19 / 05 / 2019$ 22:11 & JPG File & 588 KB \\
國 GE_HD_1920x1080.jpg & $19 / 05 / 201922: 12$ & JPG File & 1,208 KB \\
国 GE_HD_4800x2361.jpg & $19 / 05 / 2019$ 22:13 & JPG File & 3,967 KB
\end{tabular}

Figure 10. Differences in storage requirement for standard downloaded image format (e.g. screen captured).

After georeference respective images into its original coordinate (and now become a GIS spatial raster data), this spatial raster will have several different metadata details (e.g. spatial extend, projection, cell size - based on actual ground distance and others) such as in Figure 11. Unfortunately, storing GIS spatial raster/image with different resolutions are a lot more deficult and high-storage consumption than storing standard image format with different resolutions. Table 2 shows storage cost for respective spatial resolution.

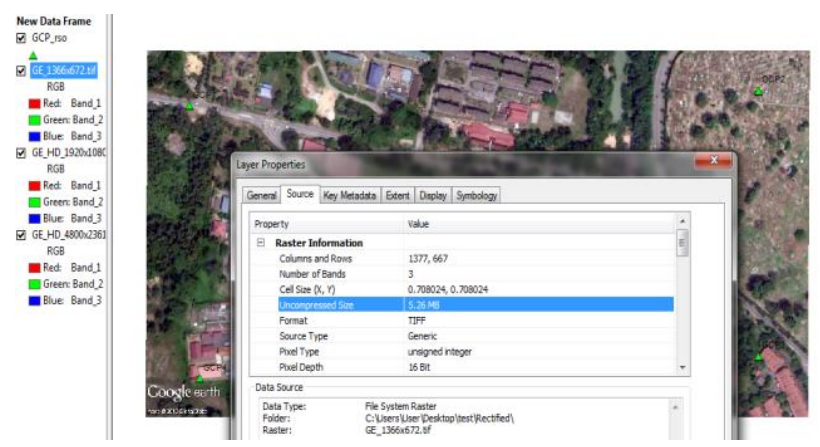

Figure 11. Metadata of the referenced photo (georeferencing)

Table 2. Differences between sample resolutions

\begin{tabular}{|c|c|c|c|}
\hline \multirow{2}{*}{$\begin{array}{l}\text { Different } \\
\text { properties }\end{array}$} & \multicolumn{3}{|c|}{ Resolution } \\
\hline & $1366 \times 672$ & $1920 \times 1080$ & $4800 \times 2361$ \\
\hline Columns/rows & 1377,667 & 1931,1074 & 4833,2345 \\
\hline Cell Size & 0.708024 & 0.440268 & 0.200984 \\
\hline Storage Size & $5.26 \mathrm{Mb}$ & $11.87 \mathrm{Mb}$ & $64.85 \mathrm{Mb}$ \\
\hline $\begin{array}{l}\text { Storage } \\
\text { increment from } \\
\text { standard format }\end{array}$ & $895 \%$ & $983 \%$ & $1,635 \%$ \\
\hline
\end{tabular}


This sample indicates that not only GIS spatial raster/imagery will contribute for the increment of storage cost (minimum 9 times of normal image format storage cost), but it also will increase as the resolution of the image increases. Thus, different image/raster resolution will produce different increment ratio in spatial raster storage cost.

As for the result from this storage cost, different quality / better sharpness at ground control with higher resolution spatial raster imagery could be obtained such as in Figure 12. This result is valid for those photo/imagery/scanned map (descrete type) of spatial raster dataset.

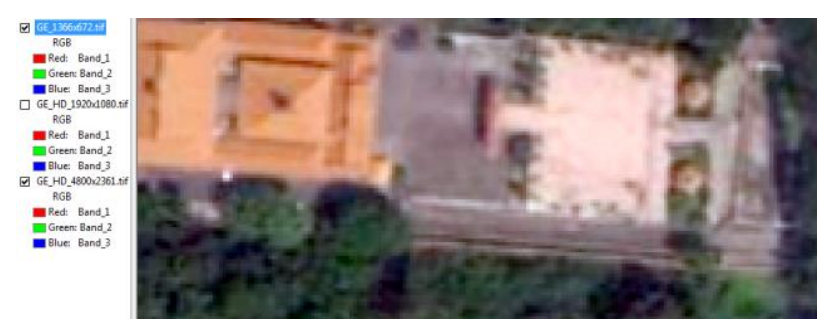

Figure 12a. Minimum resolution (1366 x 672) - blurred image

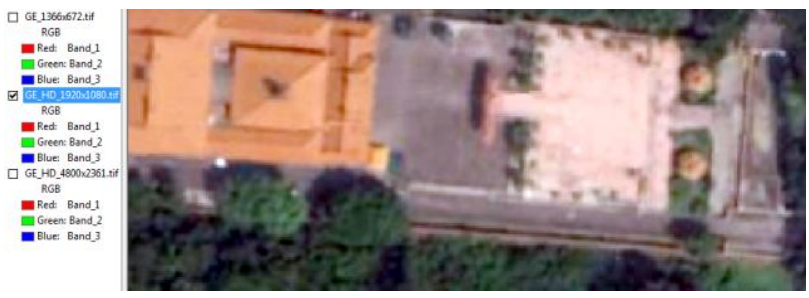

Figure 12b. HD (1920 x 1080) -clearer image compared minimum resolution -

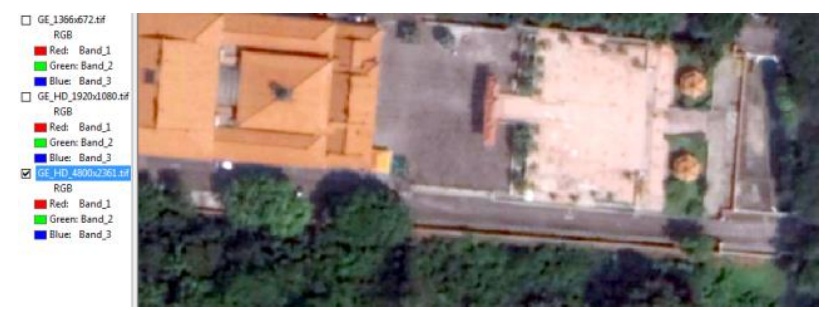

Figure 12c. Maximum resolution (4800 x 2361) - Clearest image

The test sample indicates that spatial raster data type is also carries Level of Details (LoD) which presented in its resolution properties. The detailed level (maximum resolution/pixels per inch) produces clearer and sharper image (more accurate data value) as compared to the medium and low resolution levels. However, major drawback on this technique (storing different resolutions of certain area; also could be apply at different time series) is on storage cost (capacity).

\subsubsection{DEM (Continues Raster Type)}

A continues spatial raster (e.g. Figure 8b) is a type of raster that carries a a property/parameter which having slightly different value from its neigbour but with a continuety of the layer parameter/property (e.g. elevation, density, pattern). This type of raster can can be classify to acquire discrete raster view (for visualization) or exhange to discrete by rater operation/export format. An example of multi-scale/resolution of a continues spatial raster type as shown in Figure 13; yet the main issue is the storage cost. This example of $12 \mathrm{~m}$-resolution scale
(937.34Mb) vs $4 \mathrm{~m}$-resolution $(8.23 \mathrm{~Gb})$ with storage increment of 8.78 times as compared to scale factor $3(12 \mathrm{~m} .4 \mathrm{~m})$.

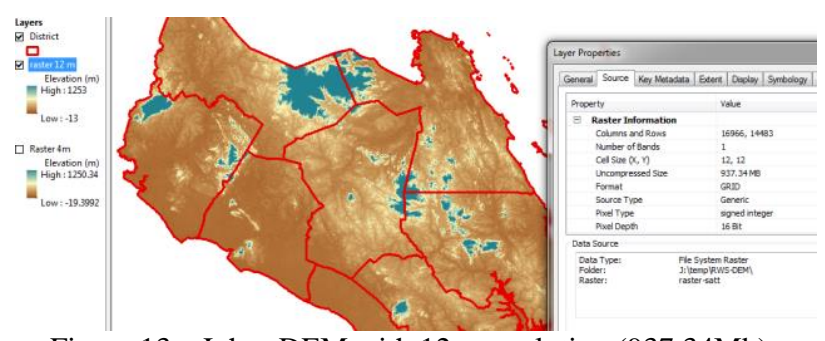

Figure 13a. Johor DEM with $12 \mathrm{~m}$ resolution $(937.34 \mathrm{Mb})$

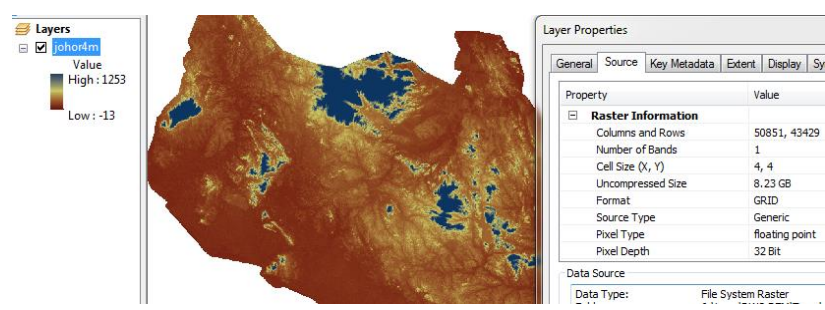

Figure $13 \mathrm{~b}$. Johor DEM with $4 \mathrm{~m}$ resolution $(8.23 \mathrm{~Gb})$

\subsection{Current Implementation}

Normal practices on raster with different resolution always come with file-based storage (folder directory) or several supported by spatial database formats such as file geodatabase from ESRI and PostgreSQL with extension PostGIS. Similarly with normal vector multi-scale implementation (storage drawback), enormous increment for storage cost (Figure 13) in storing multi-resolutions raster is more worrisome than vector multi-scale dataset.

\begin{tabular}{|c|c|c|c|}
\hline Name & Date modified & Type & Size \\
\hline [Dohor_4m_msi3.tfw & 06/05/2019 5:56 & TFW File & $1 \mathrm{~KB}$ \\
\hline 国 johor_4m_msl3.tif & 06/05/2019 6:13 & TIF File & $8,664,712 \mathrm{~KB}$ \\
\hline 星) johor_4m_msl3.tif.aux.xml & 08/05/2019 18:39 & XML Document & \\
\hline johor_4m_msl3.tif.ovr & 08/05/2019 18:39 & OVR File & $1,290,239 \mathrm{~KB}$ \\
\hline johor_4m_msl3.tif.xml & 06/05/2019 6:22 & XML Document & $1 \mathrm{KE}$ \\
\hline
\end{tabular}

Figure 13; File based consumed more storage capacity than the data properties (close to $10 \mathrm{~Gb}$ for a $4 \mathrm{~m}$ resolution raster)

Other minor drawback for multi-scale raster include but not limited to no spatial relationship between descrete raster (less in relationship for continues - chaterogized classes raster), updating problem and others.

\section{MULTI-SCALE RASTER ANALYSIS AND VALIDATION}

A raster at any resolution or detail levels can be process, analysis and validate with other relevant procedure. Example of raster processing are mosaic raster (sew a sequence of raster into larger coverage), changing the resolution, build pyramid (faster rendering time in graphic), calculation/operation on multiple raster overlay of similar resolution and others.

\subsection{Exchanging Raster Resolution}

Changing raster resolution to one another is possible by resampling method. Resampling changes the total number of pixels in the raster, which are displayed as width and height in pixels in the raster size. By increasing the number of pixels the 
dataset (up-sampling), the algorithm sub-divided pixel to be added in input raster dataset. On the other hand, down-sampling process decreases the number of pixels by merging pixel with neighbour pixels for a new low resolution raster dataset (called generalization process in vector data type). Whenever data is removed from or added to the raster, the image quality degrades to some extent (Adobe, 2016); except performing site validation procedure.

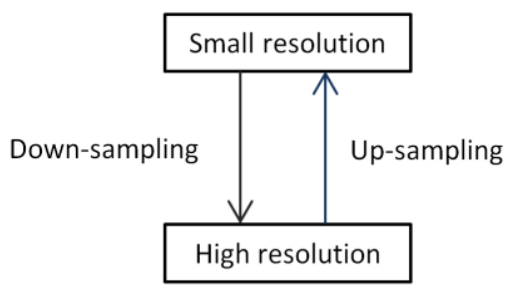

Figure 15. Resampling technique enables raster to exchange their pixel resolution.

\subsection{Raster Analysis/Processing}

Raster analysis or processing normally will consume a lot of device memory as well as processor compared to vector data type. However, it could be done much faster than vector if the pixel resolution of input rasters, covered area and projection system are both same for each input raster data (Figure 16).

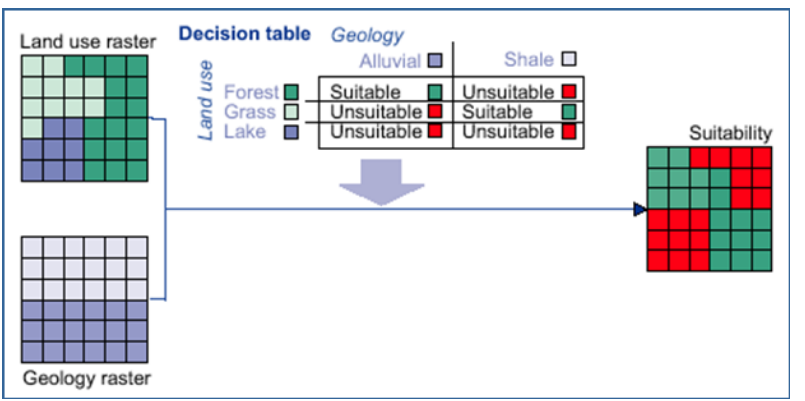

Figure 16. A typical example of raster analysis from two raster input of the same pixel size and covered area.

\subsubsection{Equal Size of Pixel Resolution}

All analysis involves two or more input raster dataset should have equal raster resolution (except to resample the raster pixel size process in a batch processing mode). Otherwise a compatible error will occured which will interupt the process.

\subsubsection{Same Coverage and Projection}

The pixel based raster analysis have more strict roles compare vector analysis. Any raster analysis (computation based on two or more input rasters) should have the same covered area of input rasters which will resulting a consistant output (without Nil-pixel value).

It is very adviceable to define the input raster projection (either in geographic coordinate system or projected coordinate system (local projection) before carry out any raster processing. New output raster (result from analysis) also should be well defined projection to have better management on raster datasets.

4.2.3 Multi-criterion Analysis - Same Years
Since the raster is made up based on pixel resolution of certain dataset - some of them from vector converted dataset. Both raster and vector type of spatial data are bounded with the date/year of it is produced. Thus, performing any analysis such as multi-criterion analysis, should consider the same time frame of the captured raster data for more realible and accurate result.

\subsection{Raster Validation}

Raster from different resolutions (multi-scale) should undergo a validation process - involves several methods/steps before and after processing take place. Some aspects to be awared before processing are on each input raster should be from the same date (month/year of collected data), site validation (e.g. features/location inspection), setting up ground control point (GCPs), error-free, and type of method used in processing (algorithm).

Validation also becoming very essential when collecting multi-scale/resolutions raster data from various source of data providers (agencies and acquisition technique (e.g. LiDAR, satellite, drone, converted from vector, scanned maps and others).

\section{CONCLUSION}

This paper discussed on the concept and current implementation of multi-scale and dimension of raster spatial dataset (although less research and term used for scale dimension in raster spatial dataset). This paper basically gives an idea on concept of multi-scale / scale dimension for GIS raster data. Some issues on storage cost and management, analysis and procedure for better analysis result. Limitation of raster such stored only one parameter is one of the biggest challenges to design a seamless scale dimension management such as vector (Figure 6 and 7).

Synergizing and incorporating multiple scale (e.g. different pixel resolutions) of single parameter raster dataset (e.g. density, elevation, image and others) will inevitably will give great contribution in rendering proses, analysis speed as well as minimizing the storage capacity (duplicate XY location for each pixel).

Next, we are looking forward a way to solve this issue as well propose solution to the current implementation and it drawbacks. Some suggestions such as utilizing spatial database could be explored near the future to define the connection between multi-scale/resolution raster of the same parameter/theme; also a raster viewer in supporting single rendering raster value at different raster resolution while preserving the quality of the dataset.

\section{REFERENCES}

Adobe, 2016. Online software tutorial https.//helpx.adobe.com/photoshop/kb/advanced-croppingresizing-resampling-photoshop.html. Access date. 19 May 2019.

GICHD IMSMA, 2016. Online wiki. http.//mwiki.gichd.org/IM/Types_of_Data. Access date. 18 May 2019. 
The International Archives of the Photogrammetry, Remote Sensing and Spatial Information Sciences, Volume XLII-4/W16, 2019 6th International Conference on Geomatics and Geospatial Technology (GGT 2019), 1-3 October 2019, Kuala Lumpur, Malaysia

Karim, H., Rahman, A. R., and Boguslawski, P., 2016.

Generalization Technique for 2D+Scale DHE Data Model.

ISPRS Volume XLII-2/W1, 2016. 3rd International

GeoAdvances Workshop, 16-17 October 2016, Istanbul,

Turkey.

Meijers, M. (2011). Variable-Scale Geo-Information. (PhD), Technische Universiteit Delft, Netherland.

Pathan, S. K, 2012. Multi Criteria Modelling in GIS. SCRIBD

Online Slide. https.//www.scribd.com/presentation/

20608630/Multi-Criteria-Modelling-in-Gis. Access date. 18

May 2019.

Pramoda Raj, 2017. Raster Data Model. Online - Slide Share. https.//www.slideshare.net/pramodgpramod/raster-data-model76694132. Access date. 18 May 2019.

Xuan Zhu, 2014. GIS and Urban Mining. Resources Journal 2014. 235-247; DOI.10.3390/resources3010235. ISSN 20799276

Revised August 2019 